\title{
Ethylene Glycol Ingestion Treated Only with Fomepizole
}

\author{
Larissa I. Velez, $M D^{a}$, Greene Shepherd, PharmD ${ }^{b}$, Yong Chan Lee, $M D^{a}$, Daniel C. Keyes, $M D^{a}$
}

aDepartment of Surgery, Division of Emergency Medicine, University of Texas Southwestern Medical Center, Dallas, TX

bUniversity of Georgia, College of Pharmacy, Athens, GA; Department of Emergency Medicine, Medical College of Georgia,

Augusta, GA

\begin{abstract}
Introduction: Ethylene glycol is a widely used chemical that is capable of causing significant injury if ingested. Treatment for ethylene glycol poisoning typically includes basic supportive care, alcohol dehydrogenase inhibition, and hemodialysis. Recent data have suggested that hemodialysis may not be necessary for cases of ethylene glycol poisoning that can be treated with fomepizole as blocking therapy before acidosis or renal dysfunction develops.

Case Report: A 33-year-old man presented to the emergency department 1 hour after drinking approximately $1 / 2$ gallon of ethylene glycol antifreeze and an unknown quantity of beer. On arrival he was mildly inebriated but otherwise displayed no other features of ethylene glycol poisoning. Fomepizole therapy was initiated and initial laboratory studies later revealed an osmol gap of 157 mOsm and an ethylene glycol concentration of $706 \mathrm{mg} / \mathrm{dL}$. Nephrology and toxicology services were consulted. Over the next 3 days, fomepizole therapy was continued while the patient's acid-base status and renal function were closely monitored. No evidence of acid-base abnormalities or renal impairment was ever observed and the patient was discharged to psychiatric care on the fourth hospital day.

Discussion: This report describes the case of a patient who presented soon after a massive ingestion of ethylene glycol with very high serum concentrations. He was successfully treated using fomepizole and basic supportive care. Our patient developed neither renal insufficiency nor metabolic acidosis. His concomitant ethanol consumption, early presentation, and treatment likely contributed to his favorable outcome. This case report underscores the effectiveness of supportive care and fomepizole in the treatment of ethylene glycol poisoning.
\end{abstract}

\section{INTRODUCTION}

Ethylene glycol is an organic solvent used as a primary agent in automobile antifreeze and other household products. Accessibility, its intoxicating effects, and its sweet taste have made it common in toxic exposures and overdoses. In 2003, 5816 exposures and 23 deaths from ethylene glycol were reported to the American Association of Poison Control Centers Toxic Exposure Surveillance System (AAPCC-TESS) [1]. Ethylene glycol overdose is characterized by central nervous system depression, metabolic acidosis, acute renal failure, and cardiopulmonary collapse. The toxicity of ethylene glycol arises from formation of metabolites that can cause severe metabolic acidosis and the formation of calcium oxalate crystals. Deposition of calcium oxalate crystals has been suggested as the primary cause of renal parenchymal damage and renal failure.

Traditionally, ethanol has been used to block alcohol dehydrogenase and, combined with hemodialysis, has been the primary modality in the care of patients poisoned by ethylene glycol. More recently, the Food and Drug Administration (FDA) has approved the use of fomepizole (4-methylpyrazole, Antizol®, Orphan Pharmaceuticals, Minnetonka, Minnesota) for the treatment of methanol and ethylene glycol poisoning. Fomepizole has since become the antidote of choice over ethanol due to its more predictable pharmacokinetics, higher affinity for alcohol dehydrogenase, ease of administration, and lack of adverse side effects

Keywords: ethylene glycol, ingestion, fomepizole, poisoning, hemodialysis

Notes: There was no outside funding of any kind for this study.

Corresponding Author: Larissa I. Velez, MD, UT Southwestern Medical Center, 5323 Harry Hines Blvd., Dallas, TX 75390-8579. Email: larissa.velez@utsouthwestern.edu 
in spite of its relatively high cost. Hemodialysis continues as the treatment of choice for massive overdoses. Current recommendations for hemodialysis in the setting of ethylene glycol poisoning are cardiopulmonary collapse, severe metabolic acidosis, severe electrolyte imbalance, renal failure, or a serum ethylene glycol level greater than $50 \mathrm{mg} / \mathrm{dL}$ [2]. We present a case of a patient who ingested ethylene glycol with an initial ethylene glycol level of $706 \mathrm{mg} / \mathrm{dL}$. He was successfully treated with fomepizole only.

\section{CASE REPORT}

A 33-year-old male with a history of depression and anxiety presented to the emergency department (ED) one hour after ingesting $\frac{1}{2}$ gallon of antifreeze and an unknown amount of ethanol. The patient stated that he kept the antifreeze "in his refrigerator, next to his beer" and accidentally drank some. Later, he admitted to drinking the antifreeze to harm himself. This was his third suicide attempt. On arrival his vital signs showed a blood pressure of $132 / 87 \mathrm{~mm} \mathrm{Hg}$, a heart rate of 95 beats per minute, a respiratory rate of 18 breaths per minute, a temperature of $36.3^{\circ} \mathrm{C}$, and a pulse oximetry of $96 \%$ on room air. He was mildly intoxicated but alert, oriented, and cooperative. He denied any complaints. His physical examination demonstrated an apparently healthy male in no apparent distress. His examination was otherwise unremarkable.

His laboratory findings showed a $\mathrm{Na}$ of $140 \mathrm{mEq} / \mathrm{L}, \mathrm{K}$ of $3.7 \mathrm{mEq} / \mathrm{L}, \mathrm{Cl}$ of $103 \mathrm{mEq} / \mathrm{L}, \mathrm{CO}_{2}$ of $27 \mathrm{mEq} / \mathrm{L}, \mathrm{BUN}$ of $8 \mathrm{mg} / \mathrm{dL}$, and creatinine of $0.9 \mathrm{mg} / \mathrm{dL}$. Anion gap was calculated as 10 . Arterial blood gases on room air showed a $\mathrm{pH}$ of $7.36, \mathrm{pCO}_{2}$ of $51 \mathrm{mmHg}$, and $\mathrm{pO}_{2}$ of $84 \mathrm{mmHg}$. He had a measured osmolality of 446 mOsm, a calculated osmolality of 289 mOsm, and an osmolar gap of 157. The serum ethanol concentration was $84 \mathrm{mg} / \mathrm{dL}$ with an initial ethylene glycol level of $706 \mathrm{mg} / \mathrm{dL}$ (measured by gas chromatography, LabCorp). Urinalysis demonstrated a specific gravity of 1.010 and was negative for crystals.

Intravenous fomepizole therapy was initiated in the ED 4 hours after ingestion with a loading dose of $15 \mathrm{mg} / \mathrm{kg}$. Fomepizole was continued every 12 hours for a total of 8 doses $(10 \mathrm{mg} / \mathrm{kg}$
IV $\mathrm{x} 4$ doses then $15 \mathrm{mg} / \mathrm{kg}$ IV $\mathrm{x} 4$ doses) until the ethylene glycol level was $6 \mathrm{mg} / \mathrm{dL}$ and the osmolar gap was $7 \mathrm{mOsm}$. The toxicology service recommended hemodialysis because of the elevated ethylene glycol level; instead, the nephrology service observed the patient because of a normal acid-base status and continued inhibition of ADH. The patient was closely monitored over the next 3 days. Table 1 lists available laboratory values collected during the patient's hospital stay. As the osmolar gap and ethylene glycol level decreased, the patient's renal function remained normal. The bicarbonate levels also remained stable, demonstrating that the patient did not develop a metabolic acidosis. He was discharged to a psychiatric hospital on hospital day number 4 .

\section{DISCUSSION}

Ethylene glycol is a water-soluble alcohol that is quickly absorbed by the gastrointestinal (GI) tract and has a volume of distribution of $0.5-0.8 \mathrm{~L} / \mathrm{kg}$ [2]. The liver metabolizes ethylene glycol via alcohol dehydrogenase and aldehyde dehydrogenase to acidic compounds that are responsible for the metabolic acidosis and the renal failure. Unmetabolized ethylene glycol is excreted through the kidneys without causing injury. The half-life of the ethylene glycol is approximately 3 hours in the absence of treatment or coingested ethanol, but the metabolites can remain for several days.

\section{CLINICAL FEATURES}

Ethylene glycol toxicity is initially characterized by central nervous system (CNS) depression and followed by a high anion gap metabolic acidosis. In severe overdoses cardiopulmonary collapse, hypocalcemia, and renal failure ensue. Its initial presentation to the treating clinician depends on the time elapsed since the ingestion, co-ingestion of alcohol dehydrogenase inhibitors, and amount ingested.

The definitive laboratory test for ethylene glycol poisoning is an ethylene glycol serum concentration, but this can be difficult to acquire for some laboratories. The combination of an osmolar

\begin{tabular}{|c|c|c|c|c|c|c|c|c|}
\hline Date/time & $\begin{array}{c}\text { Anion gap } \\
(\mathrm{mmol} / \mathrm{L})\end{array}$ & $\begin{array}{c}\text { Bicarbonate } \\
(\mathbf{m m o l} / \mathrm{L})\end{array}$ & $\begin{array}{c}\text { BUN } \\
(\mathbf{m g} / \mathbf{d l})\end{array}$ & $\begin{array}{c}\text { creatinine } \\
(\mathbf{m g} / \mathbf{d l})\end{array}$ & $\begin{array}{c}\text { Osmolar } \\
\text { gap }\end{array}$ & $\begin{array}{c}\text { EtOH } \\
(\mathbf{m g} / \mathrm{dl})\end{array}$ & pH & $\begin{array}{l}\text { EG level } \\
(\mathbf{m g} / \mathbf{d l})\end{array}$ \\
\hline $12 / 02 / 00,0050$ & 10 & 27 & 8 & 0.9 & 157 & 84 & 7.36 & 706 \\
\hline $12 / 02 / 00,0700$ & 7 & 25 & & & & & 7.39 & \\
\hline $12 / 02 / 00,1850$ & 8 & 30 & & & & & & \\
\hline $12 / 03 / 00,0410$ & 6 & 28 & 6 & 0.9 & & & & 200 \\
\hline $12 / 03 / 00,1220$ & 7 & 28 & 7 & 0.9 & 35 & & & \\
\hline $12 / 04 / 00,0400$ & 9 & 26 & 13 & 1 & 13 & & & 48 \\
\hline $12 / 05 / 00,0400$ & 9 & 27 & 11 & 1 & 7 & & & 6 \\
\hline $12 / 06 / 00,0400$ & 10 & 26 & 12 & 0.9 & 6 & & & 0 \\
\hline
\end{tabular}


gap with an anion gap acidosis is still the most common way that an ethylene glycol or methanol ingestion is suspected, but the validity of this approach has been challenged [3]. The generally accepted minimum dose to cause any form of toxicity is $1 \mathrm{mg} / \mathrm{kg}$, which correlates to a peak serum concentration of approximately $20 \mathrm{mg} / \mathrm{dL}$. Specific therapeutic interventions should be based on a combination of history, clinical presentation, and measured concentrations. The initial osmolar gap is due to the presence of the parent compound, ethylene glycol. Therefore, this osmolar gap is transient. With regard to the anion gap, the opposite is true since the metabolites are primarily responsible for this anion gap. As metabolism progresses after ingestion, the osmolar gap will decrease and the anion gap increases-unless timely antidotal therapy is administered.

As the concentration of oxalate increases, calcium oxalate crystal formation is rapid and, in severe cases, can result in systemic hypocalcemia. Deposition of crystals in soft tissue can be very painful and lead to dysfunction of organs. Monohydrate and dihydrate crystals can usually be seen in the urinalysis 4-8 hours after ingestion and persist for approximately 2 days; however, since crystals are not seen in all cases, their absence cannot rule out ethylene glycol poisoning [4].

The initial treatment should be directed at basic supportive measures. GI decontamination by gastric emptying or the use of activated charcoal has limited utility. Antidotal therapy of ethylene glycol poisoning is usually directed to blocking the formation of toxic metabolites. The traditional mainstay for antidotal treatment has been ethanol since Wacker published his study in 1965 [5]. Ethanol competitively binds alcohol dehydrogenase to prevent the metabolism of ethylene glycol. In our patient the coingestion of ethanol may have contributed to his benign outcome. The disadvantage to the routine use of ethanol as an antidote is the associated CNS depression, gastrites, hepatic toxicity, and the potential for hypoglycemia. Maintaining a therapeutic level is often difficult because of the need for frequent serum monitoring and dosing adjustments. Given these challenges, hemodialysis has been recommended for patients with an initial ethylene glycol level $>50 \mathrm{mg} / \mathrm{dL}$. Other indications that are often cited for dialysis include severe metabolic acidosis, cardiopulmonary collapse, and renal failure [6].

In 1986, fomepizole was introduced as an effective inhibitor of alcohol dehydrogenase [5,7]. In 1997, fomepizole received FDA approval for the treatment of ethylene glycol poisoning in adults. The indications for fomepizole use are similar to indications for ethanol treatment [2]. Indications for fomepizole include: an ethylene glycol concentration $>20 \mathrm{mg} / \mathrm{dL}$; a recent history of ingesting toxic amounts of ethylene glycol and osmolar gap $>10 \mathrm{mosm} / \mathrm{L}$; or a history or strong clinical suspicion of ethylene glycol poisoning, and at least two of the following criteria: arterial $\mathrm{pH}<7.3$ serum bicarbonate $<20 \mathrm{mEq} / \mathrm{L}$, osmolar gap $>10 \mathrm{mosm} / \mathrm{L}$, or urinary oxalate crystals in urine. The dosing regimen for fomepizole is $15 \mathrm{mg} / \mathrm{kg}$ IV followed by $10 \mathrm{mg} / \mathrm{kg}$ IV every 12 hours for 4 doses. After the aforementioned dosing, the dose is increased to $15 \mathrm{mg} / \mathrm{kg}$ every 12 hours until a plasma concentration of ethylene glycol goes below $20 \mathrm{mg} / \mathrm{dL}$. Since fomepizole is dialyzable, patients undergoing concomitant hemodialysis have the dosing interval reduced to every 4 hours.

Brent et al. published the Methylpyrazole for the Treatment of Toxic Alcohol (META) study supporting the use of fomepizole for ethylene glycol poisoning [4]. The META study demonstrated that fomepizole could be used effectively in the treatment of ethylene glycol intoxication. The highest serum concentration of ethylene glycol documented in this study was $446 \mathrm{mg} / \mathrm{dL}$ in a patient with an initially normal creatinine. The authors concluded that hemodialysis is probably not required in patients without acidosis or renal dysfunction [4]. Sivilotti, in his follow up analysis of the META study, states that patients treated with fomepizole only need hemodialysis in the presence of metabolic acidosis or abnormal renal function [8]. Several case reports and case series have supported this premise. Hantson presented a case of a 19-year-old female who presented within an hour of an ethylene glycol ingestion. Her initial ethylene glycol level was $160 \mathrm{mg} / \mathrm{dL}$, and she was successfully treated with fomepizole alone [9]. Najafi presented a recent case with an initial ethylene glycol level of $202 \mathrm{mg} / \mathrm{dL}$ with a $\mathrm{pH}$ of 7.17 . This patient was also successfully treated with fomepizole alone [10]. Baud presented 4 cases of patients with ethylene glycol concentrations as high as $320 \mathrm{mg} / \mathrm{dL}$ and $\mathrm{pH}$ as low as 7.12 that were safely treated with fomepizole alone [11]. Borron presented a series of 11 patients with ethylene glycol toxicity of which 8 were successfully treated with fomepizole alone [12]. Boyer and Harry each presented two pediatric cases with similar results $[13,14]$. The highest ethylene glycol level in a patient who was treated with fomepizole without hemodialysis comes from a Norwegian case report that detailed an initial ethylene glycol concentration of $585 \mathrm{mg} / \mathrm{dL}$ [15].

Our case is the highest reported ethylene glycol concentration successfully treated with fomepizole and supportive care alone. Our patient had also been drinking ethanol at the time of the overdose, and the ethanol likely contributed to the magnitude of his ethylene glycol concentration at presentation since its metabolism would have been inhibited. This probably played a role in his positive outcome. The calculated half-life of ethylene glycol in our patient was 11 to 13 hours, which is faster than the 17-18 hour half-life during fomepizole treatment as reported by Sivilotti [8]. Although this implies that ADH may not have been as completely inhibited at such a high serum concentration of ethylene glycol, fomepizole monotherapy was very clinically effective in our patient. Furthermore, the duration of fomepizole therapy is probably less than previously described.

There have been higher concentrations reported in patients treated with fomepizole in combination with hemodialysis. Davis and Curtin reported concentrations of $888 \mathrm{mg} / \mathrm{dL}$ and $905 \mathrm{mg} / \mathrm{dL}$ respectively [16,17]. Johnson in 1999 reported a record high concentration of $1889 \mathrm{mg} / \mathrm{dl}$ in a patient who ingested an estimated 3L of ethylene glycol [18]. Again, time of presentation post-ingestion and the degree of acidemia and renal dysfunction seem more important than the magnitude of blood concentrations in predicting the outcomes of treated patients. 


\section{CONCLUSION}

This report describes the case of a patient who presented soon after a massive ingestion of ethylene glycol with very high serum concentrations. He was successfully treated using standard doses of fomepizole over three days and basic supportive care. Our patient did not develop renal insufficiency or metabolic acidosis. His early presentation and concomitant ethanol consumption likely contributed to his favorable outcome, obviating the need for hemodialysis. This case underscores the effectiveness of fomepizole and supportive care in the treatment of ethylene glycol poisoning, even at levels that have traditionally required hemodialysis.

The authors have no potential financial conflicts of interest to report.

\section{REFERENCES}

1. Watson WA, Litovitz TL, Klein-Schwartz W, et al. 2003 annual report of the American Association of Poison Control Centers Toxic Exposure Surveillance System. Am J Emerg Med 2004;22(5):335-404.

2. Barceloux DG, Krenzelok EP, Olson K, et al. American Academy of Clinical Toxicology practice guidelines on the treatment of ethylene glycol poisoning. Ad hoc committee. J Toxicol Clin Toxicol. 1999;37(5):537-560.

3. Glaser DS. Utility of the serum osmol gap in the diagnosis of methanol or ethylene glycol ingestion. Ann Emerg Med.

1996;27(3):343-346.

4. Brent J, McMartin K, Phillips S, et al. Fomepizole for the treatment of ethylene glycol poisoning. Methylpyrazole for Toxic Alcohols Study Group. N Engl J Med. 1999;340(11):832-838.

5. Anonymous. Ethylene glycol antidote cleared for marketing. (News) Am J Health Syst Pharm. 1998;55(2):110.

6. Joliff H, Sivilotti M. Ethylene glycol. In: Dart RC, editor. Medical Toxicology. Philadelphia: Lippincott Williams \& Wilkins; 2004. pp. 1229-1230.
7. Baud FJ, Bismuth C, Garnier R, et al. 4-Methylpyrazole may be an alternative to ethanol therapy for ethylene glycol intoxication in man. J Toxicol Clin Toxicol. 1986;24(6):463-483.

8. Sivilotti ML, Burns MJ, McMartin KE, et al. Toxicokinetics of ethylene glycol during fomepizole therapy: Implications for management. For the Methylpyrazole for Toxic Alcohols Study Group. Ann Emerg Med. 2000;36(2):114-125.

9. Hantson P, Hassoun A, Mahieu P. Ethylene glycol poisoning treated by intravenous 4-methylpyrazole. Intensive Care Med. 1998;24(7):736-739.

10. Najafi CC, Hertko LJ, Leikin JB, et al. Fomepizole in ethylene glycol intoxication. Ann Emerg Med. 2001;37(3): 358-359.

11. Baud FJ, Galliot M, Astier A, et al. Treatment of ethylene glycol poisoning with intravenous 4-methylpyrazole. $N$ Engl J Med. 1988;319(2):97-100.

12. Borron SW, Megarbane B, Baud FJ. Fomepizole in treatment of uncomplicated ethylene glycol poisoning. Lancet. 1999;354(9181):831.

13. Boyer EW, Mejia M, Woolf A, et al. Severe ethylene glycol ingestion treated without hemodialysis. Pediatrics.

2001; 107(1):172-173.

14. Harry P, Jobard E, Briand M, et al. Ethylene glycol poisoning in a child treated with 4-methylpyrazole. Pediatrics. 1998;102(3):E31.

15. Aakervik O, Svendsen J, Jacobsen D. [Severe ethylene glycol poisoning treated with fomepizole (4-methylpyrazole)].

Tidsskr Nor Laegeforen. 2002;122(25):2444-2446.

16. Davis DP, Bramwell KJ, Hamilton RS, et al. Ethylene glycol poisoning: Case report of a record-high level and a review.

J Emerg Med. 1997;15(5):653-667.

17. Curtin L, Kraner J, Wine H, et al. Complete recovery after massive ethylene glycol ingestion. Arch Intern Med.

1992;152(6):1311-1313.

18. Johnson B, Meggs WJ, Bentzel CJ. Emergency department hemodialysis in a case of severe ethylene glycol poisoning. Ann Emerg Med. 1999;33(1):108-110. 